\title{
A life devoted to fossil vertebrates and the collections of the Museum für Naturkunde in Berlin - Wolf-Dieter Heinrich: contributions to palaeontology
}

\author{
Lutz Christian Maul ${ }^{*}, 1$, Gottfried Böhme ${ }^{* *, 2}$, Oliver Hampe H**, $^{* *}$, Stephan Schultka Hex*, $^{*}$ \\ \& Anthony J. Stuart $* * * * *, 3$ \\ ${ }^{1}$ Forschungsstation für Quartärpaläontologie Weimar, Forschungsinstitut und Naturmuseum Senckenberg, \\ Am Jakobskirchhof 4, D-99423 Weimar, Germany \\ 2 Museum für Naturkunde der Humboldt-Universität zu Berlin, Invalidenstraße 43, D-10115 Berlin, Germany \\ ${ }^{3}$ Department of Biology, University College, Gower Street, London, WC1E 6BT, and University of Durham, \\ School of Biological and Biomedical Sciences, South Road, Durham DH1 3LE, United Kingdom
}

Received 18 September 2006, accepted 20 September 2006

Published online 30 January 2007

With 4 figures

On the occasion of his $65^{\text {th }}$ birthday, the celebrated Berlin vertebrate palaeontologist Wolf-Dieter Heinrich can look back on a very successful and creative scientific life. His research fields cover (in taxonomic order) fossil fishes, reptiles, birds, and Mesozoic mammals, and especially Quaternary mammals, large and small. Moreover, his wide interests also include fossil invertebrates, and interdisciplinary issues in geo- and biosciences (see list of publications). However, his most important contribution, for which he has achieved a well-deserved international reputation, has been in the field of Pliocene and Pleistocene small mammals.

Wolf-Dieter Heinrich was born on September 20, 1941 in Potsdam, where he attended school from 1948 until his matriculation in 1960. His biology teacher encouraged him to collect fossils from the glacial drift, igniting his interest in palaeontology. Before beginning his university study in 1960/61 he worked as a scientific volunteer at the Central Geological Institute in Berlin, where he was mainly dealing with microfossil processing. In August 1961 he enrolled for Geology at the Humboldt University in Berlin where he first discovered his interest in vertebrate palaeontology. Wolf-Dieter Heinrich agreed to work on Miocene otoliths from Hohen Woos in South-west Mecklenburg (North-eastern Germany), under the supervision of the late Kurt
Diebel (a micropalaeontologist in the Museum für Naturkunde Berlin).

This study was of such a high scientific standard that in 1967 it was accepted as a doctoral dissertation. After obtaining his doctor degree in 1966/67, a habilitation canditature followed in 1967-1972. In 1973 he was employed as Scientific Assistant in the Exhibition Department of the Museum für Naturkunde in Berlin. From September 1, 1976 he became Curator of the fossil fishes, amphibians and reptiles in the department 'Paläontologisches Museum', and received his habilitation in 1979. In 1993 he became Head Curator for all fossil vertebrate collections in the re-founded 'Institut für Paläontologie' in the Museum für Naturkunde. After restructuring in 1997 he became responsible for the fossil mammal collection.

The event that most influenced the specific focus of his palaeontological research was the discovery of the Late Pleistocene/Early Holocene fossil animal burrows at Pisede near Malchin (Mecklenburg, North-eastern Germany). This was the first major Quaternary site to be discovered in the Young Moraine region of North-east Germany. Following preliminary surveys of the site in 1966, systematic excavations were made from 1968 directed by K. Diebel, and from 1971 by W.-D. Heinrich. The site was extremely rich in small vertebrate remains.

\footnotetext{
* Corresponding author: e-mail: Imaul@senckenberg.de

** E-mail: gottfried.boehme@museum.hu-berlin.de

*** E-mail: oliver.hampe@museum.hu-berlin.de

**** E-mail: stephan.schultka@ museum.hu-berlin.de

***** E-mail: tony@megafauna.freeserve.co.uk
} 
His investigations of the micromammals from this site became the starting point of work on this biostratigraphically important fossil group, which has continued to the present day. The overall coordination of the Pisede excavation and the resulting monographs which he edited (3 volumes: 1975, 1977, 1983) were very important in establishing his scientific reputation.

At this time, the Hungarian palaeontologists Miklos Kretzoi (1907-2005) and Dénes Jánossy (1926-2005), together with Oldřich Fejfar from Prague, were among the leading international authorities in Quaternary small mammal research. They worked in close scientific collaboration with Hans-Dietrich Kahlke, the founder of the Weimar Institute of Quaternary Palaeontology. From scientific exchanges with M. Kretzoi and D. Jánossy, several research visits to Budapest, participation in excavations of Hungarian fossil sites, and last but not least his participation in the International Palaeontological Colloquia in Weimar (1968, 1972, 1985) organised by H.-D. Kahlke, Wolf-Dieter Heinrich also became an internationally recognized specialist in Quaternary micromammals. In collaboration with D. Jánossy he then described the fossil material of small mammals and birds from the classic Thuringian (Central German) Pleistocene sites Taubach (1977) and Burgtonna (1978).

In 1971/72 Wolf-Dieter Heinrich made his first contribution of supraregional importance, in his work on the Burgtonna small mammal remains. In the upper part of the profile ('Deckschichtenprofil') overlying the well-known travertine deposit he distinguished two small mammal faunas of different ages. The younger one yielded the first Pleistocene record of the steppe lemming Lagurus lagurus in Central Germany from an open-air site. This made possible an interregional correlation of the Late Pleistocene Lagurus immigration event.

In addition to the sites in his home area of Brandenburg, such as Buchow-Karpzow, Ketzin, (the Late Pleistocene so-called 'Rixdorf Horizon'), Wolf-Dieter Heinrich focussed on Thuringian Pleistocene sites, in particular the small mammal remains. In his habilitation thesis, which he defended in 1979, he investigated the palaeontology and stratigraphy of various Quaternary sites from Thuringia, and from 1979 several papers followed on the hominid sites Bilzingsleben, WeimarEhringsdorf and Weimar-Taubach. In 1982 he wrote a preliminary report about the small mammals of the Late Early Pleistocene locality Untermaßfeld, and in 1984 he published the small mammal remains from the Park Cave in Weimar.

The name of Heinrich will be forever associated with the SDQ index ('Schmelzbanddifferenzierungsquotient' - in English - relative enamel thicknesses quotient), which he devised in 1978 for the water vole genus Arvicola. Evolutionary changes in the enamel thickness in Arvicola were observed half a century ago by Hinton and Heller, and also used broadly for taxonomic and stratigraphic discri-

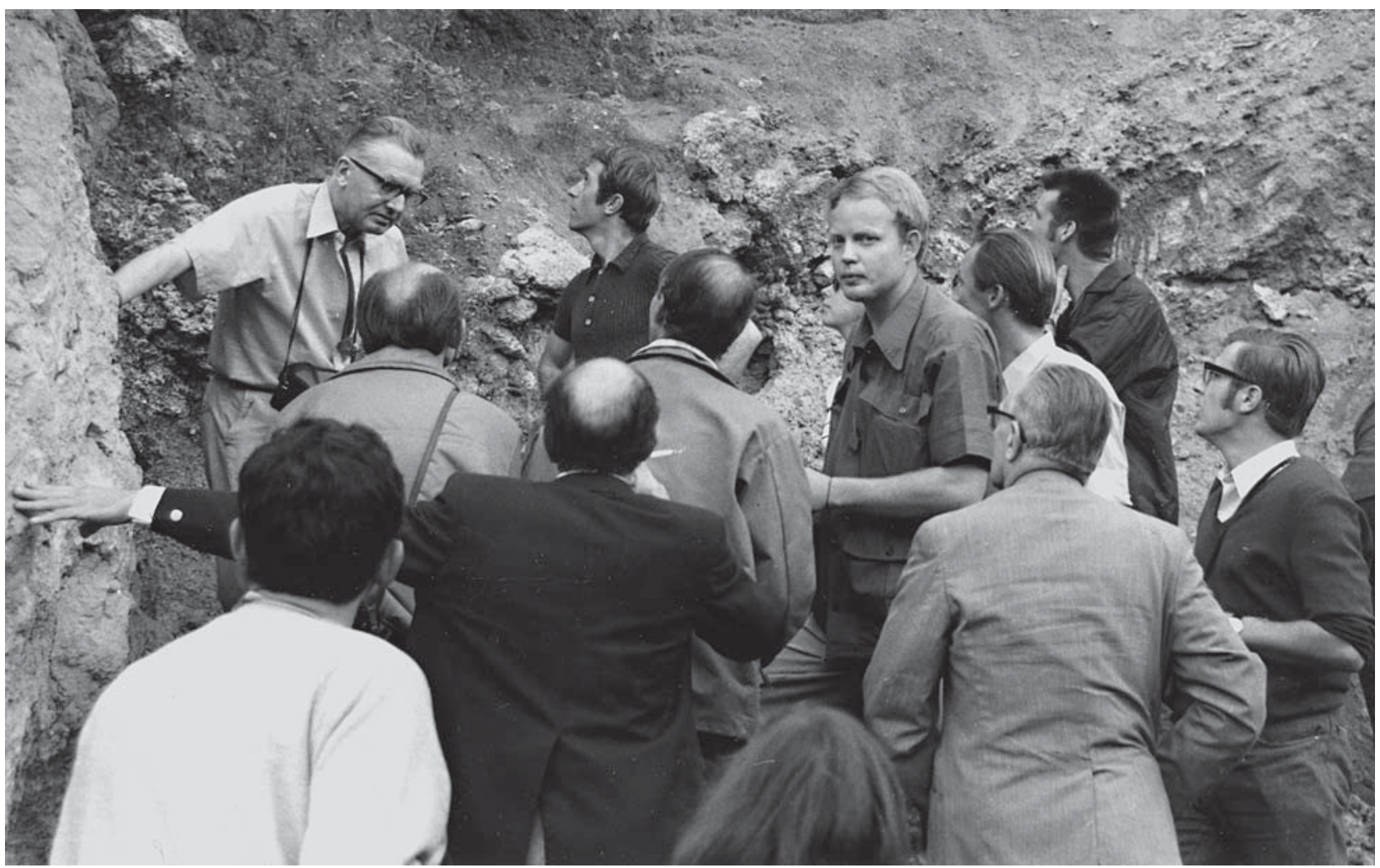

Fig. 1. Wolf-Dieter Heinrich looking into the camera at the Pleistocene section in Burgtonna during the III International Palaeontological Colloquium in Weimar 1972 (photograph FIS, Weimar). 
mination. However, the quantification of this feature, by means of the SDQ, allowed very precise tracking of the progressive evolution of the water vole lineage, allowing determination of the precise stratigraphic order of fossil Arvicola samples, and thus the entire fossil assemblages. Today when assessing the stratigraphic position of any Quaternary assemblage, this approach has become standard, ensuring W.-D. Heinrich's key international standing in the development of modern Quaternary biostratigraphic methods. Moreover, his careful quantitative analysis of changes in the water vole lineage has pointed the way to subsequent studies of evolution in the Quaternary fossil record.

Wolf-Dieter Heinrich noted, that the SDQ values of the Arvicola sample from the Lower Travertine of Weimar-Ehringsdorf differed markedly from what one would expect from an Eemian Interglacial locality. This was the first indication that the age traditionally assigned to this famous site was incorrect. Since then detailed revision of several fossil groups confirmed the pre-Eemian age of Ehringsdorf, first proposed by Heinrich.

Micromammals, in particular voles (arvicolids) are generally the best key fossils in Pliocene and Pleistocene sites. On this basis, from 1938 M. Kretzoi started to develop a biostratigraphic subdivision for the Pannonian Basin (Hungary). However, the scheme was always strongly influenced by the palaeoecological particularities of this region. In collaboration with a colleague from Prague, O. Fejfar, in 1980 Wolf-Dieter Heinrich proposed a new stratigraphic subdivision of European Pliocene and Pleistocene small mammal faunas. The stratigraphic boundaries were defined by the first and last occurrence of arvicolid taxa (Microtus, Arvicola, Mimomys etc.), which were widespread across the whole of Europe, thus allowing geographically far-ranging correlations. Today the Fejfar \& Heinrich scheme, with frequent updates (1980, 1981, 1987, 1990, 1998, etc.), is widely accepted as an international standard.

To celebrate the $100^{\text {th }}$ birthday of the important German vertebrate palaeontologist Wilhelm Otto Dietrich, who worked for many years in the $\mathrm{Mu}-$ seum für Naturkunde in Berlin, Wolf-Dieter Heinrich organised an international conference on vertebrate palaeontology and stratigraphy. $\mathrm{He}$ also edited the two volumes of proceedings that resulted from this conference. In 1987, together with O. Fejfar he organised another conference in Rohanov (Czech Republic), as the first "International Symposium on Evolution, Phylogeny and Biostratigraphy of Arvicolids (Rodentia, Mammalia)", in which 33 small-mammal palaeontologists from 14 countries presented new results on fossil arvicolids. Wolf-Dieter Heinrich was co-editor of the proceedings volume. Moreover, he was organiser of the $62^{\text {th }}$ annual conference of the Paläontologische $\mathrm{Ge}$ sellschaft 1992 in Berlin.

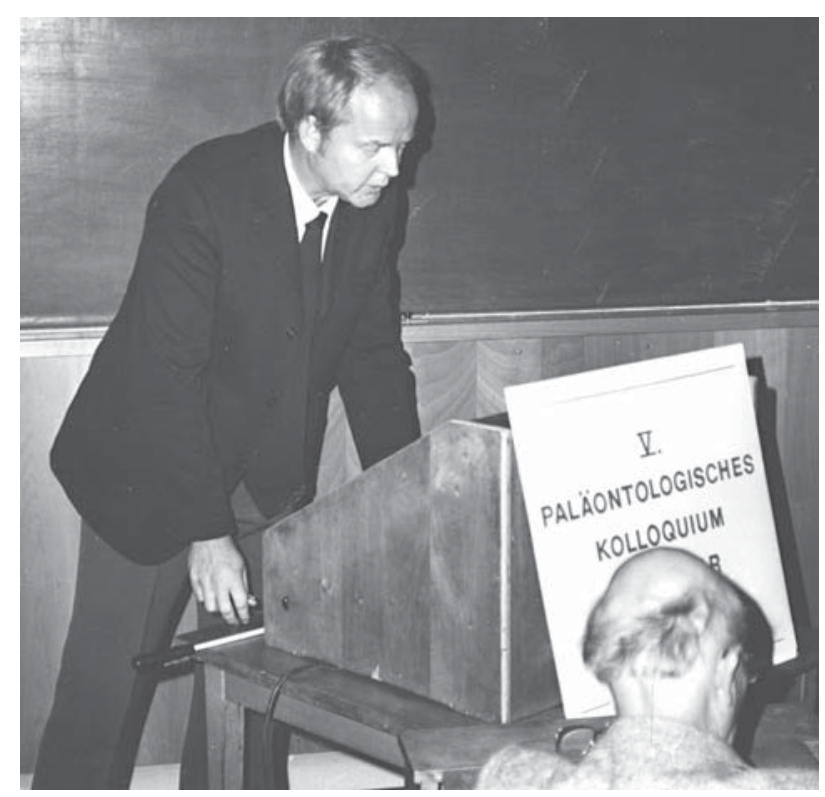

Fig. 2. W.-D. Heinrich presenting a paper at the $\mathrm{V}$ International Palaeontological Colloquium in Weimar 1985 (photograph FIS, Weimar).

With several publications about evolutionary trends in beaver teeth (Castor and Trogontherium) since 1989, Wolf-Dieter Heinrich was able to establish new methods for determining the relative age of Quaternary sites. This work is important as it allows an independent cross-checking of the results from the arvicolid investigations. These methods were first applied to the material from the fossil sites of Voigtstedt, Süßenborn, Bilzingsleben, Ehringsdorf, and Taubach and confirmed the arguments for their biostratigraphic age.

From 1990, his work on further small mammal faunas from Central German sites, such as Gamsenberg, Neumark-Nord, Gröbern, Grabschütz, Schönfeld, etc. is significantly adding to the biostratigraphic database of Central European sites.

A further period in Wolf-Dieter Heinrich's scientific life began, when he was inspired by the fossil mammal remains from the Late Jurassic Tendaguru Beds in East Africa (at the same time the oldest record of a mammal on this continent) to give more intensive attention to the results of the German Tendaguru-Expedition of 1909-1913, and with the dinosaur collection of the Museum für Naturkunde.

Meticulously, and with considerable persistence Wolf-Dieter Heinrich processed the sediment samples that still remained in the museum from the 1909-1913 excavations. He soon found fossils and obtained further records of Late Jurassic mammals. In autumn 1989, during the historic days of German re-unification, a complete preparation of the specimen, described in 1927 by W. O. Dietrich as Brancatherulum tendagurense, was made by the senior preparator H.-H. Krueger. Following this W.-D. Heinrich made $\mathrm{x}$-ray photographs in the 
Charite hospital of the mandible and published a revised description. These investigations allowed a much more precise reclassification of this important find. Originally, referred to the Pantotheria, Brancatherulum tendagurense was later assigned to the Eupantotheria, Amphitheriidae, and Paurodontidae and most recently to the ?Peramuridae.

Our understanding of early mammalian evolution was crucially augmented by the research of Wolf-Dieter Heinrich. As a result of processing ca $500 \mathrm{~kg}$ of sediment matrix, Wolf-Dieter Heinrich was able to demonstrate the presence of three further mammal genera in the Middle Saurian Bed (Kimmeridgian-Tithonian): Tendagurutherium dietrichi (Eupantotheria, ?Peramuridae), Tendagurudon janenschi (Triconodonta, Familia incertae sedis), and Allostaffia aenigmatica (Allotheria, Haramyidae).

Wolf-Dieter Heinrich played a decisive role in several projects on the palaeobiology of the dinosaurs from Tendaguru. He studied original manuscripts and field sketches by Werner Janensch and published a detailed overview on the taphonomy of the finds. Wolf-Dieter Heinrich was one of the

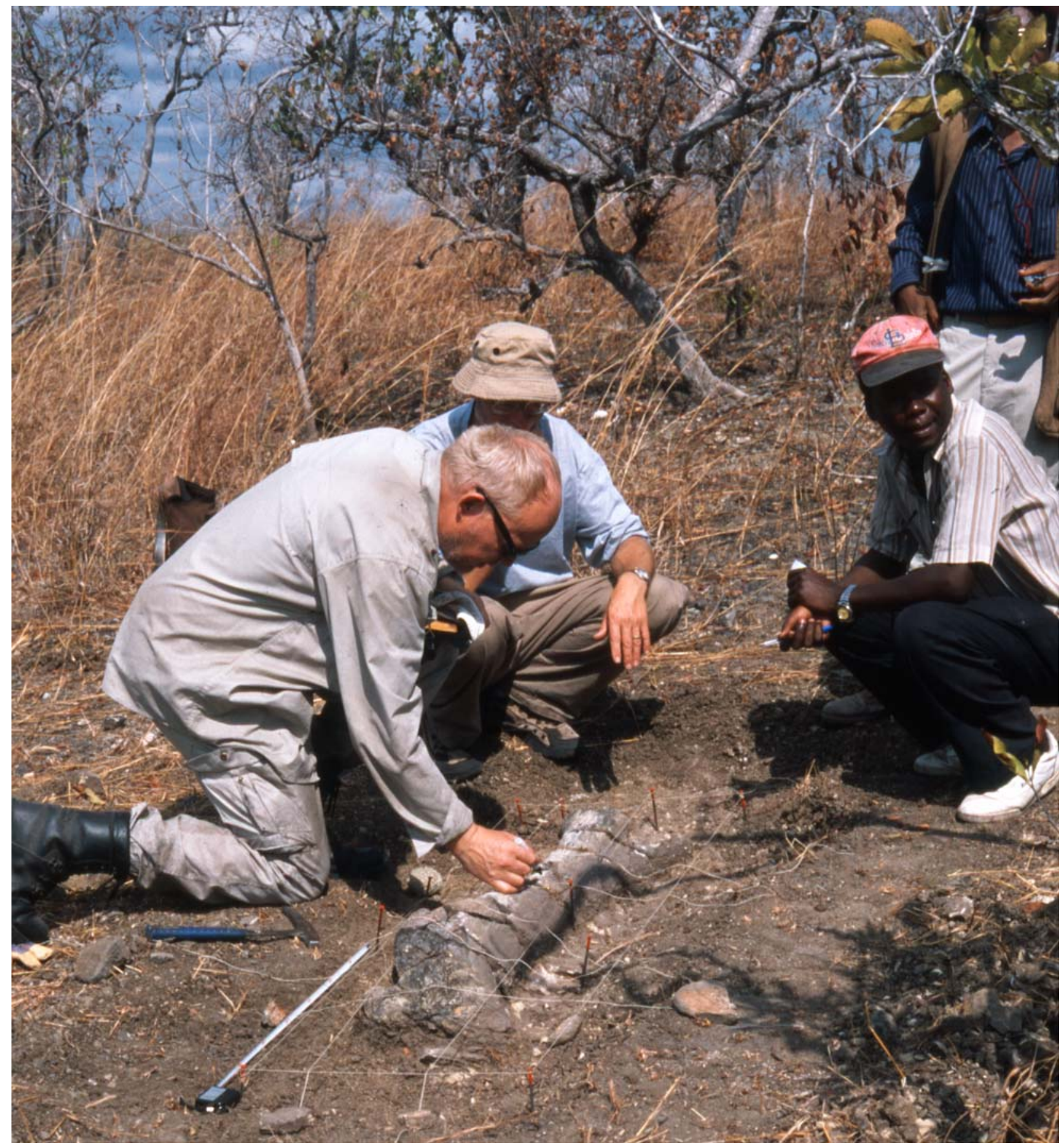

Fig. 3. W.-D. Heinrich examining a sauropod propodial with G. Maier, Calgary (behind him), and R. Chami, Dar Es Salaam (right), at the famous Tendaguru site during late summer 2000 (photograph by O. Hampe). 
initiators of the German-Tanzanian Expedition in 2000, 87 years after the conclusion of the wellknown excavations at one of the world's most important dinosaur 'Lagerstätten'. This gave him the chance to see the excavation sites of 1909-1913 with his own eyes. A wonderful feeling overcame him after his arrival in Dar Es Salaam in August 2000 when he stood at the coast of the Indian Ocean and reflected on the excellent job done by Werner Janensch, the leader of the 1909-1913 expedition, and his scientific colleagues.

At the time of his $65^{\text {th }}$ birthday Wolf-Dieter Heinrich is involved in numerous projects working at his customary level of perfection and rigour. One can safely assume that the term 'retirement' will not apply to his scientific work.

A few words about Wolf-Dieter Heinrich as a person. To a newcomer the first impression may be of someone rather withdrawn, perhaps even a little cold. Correct and precise, occasionally almost 'pernickety', he organises his work, as he organises his whole life, with the help of his wife and his colleagues. He is dogged, patient and sensible, as was evident e.g. during the big expedition to Tendaguru in 2000. Here he demonstrated that he is one of the very few people, who are able to remove the shell of a (boiled) egg without destroying the 'skin' beneath.
Entering his workroom unannounced, one feels conscious of having disturbed a 'typical scholar', who is reluctant to put aside the objects of his research, and indeed: this is the case. Many bad experiences in his long life as a scientist have made him cautious, and so he opens up only slowly to his visitor. However, then Wolf-Dieter Heinrich appears as a man who enjoys a glass of wine, who suddenly turns out to be a sensitive and openminded person, who can laugh at a corny joke as well as a sophisticated intellectual one. As he starts to talk, one is increasingly struck by how devotedly and passionately he is attached to the museum specimens for which he is responsible. It becomes apparent why he gives priority to all these 'dead' animals, which are in his care - before he turns towards the 'living', since human beings are able to help themselves.

But his love and affection are not solely directed to his research and museum collections; the $\mathrm{Mu}$ seum as a whole is also close to his heart. For many years he worked tirelessly to keep palaeontology as part of a big international museum, and to preserve its standing within the whole context. He never pushed himself to the fore, but also never avoided his responsibilities. For example, in September 1992 he took over the directorship of the department 'Paläontologisches Museum' after Her-

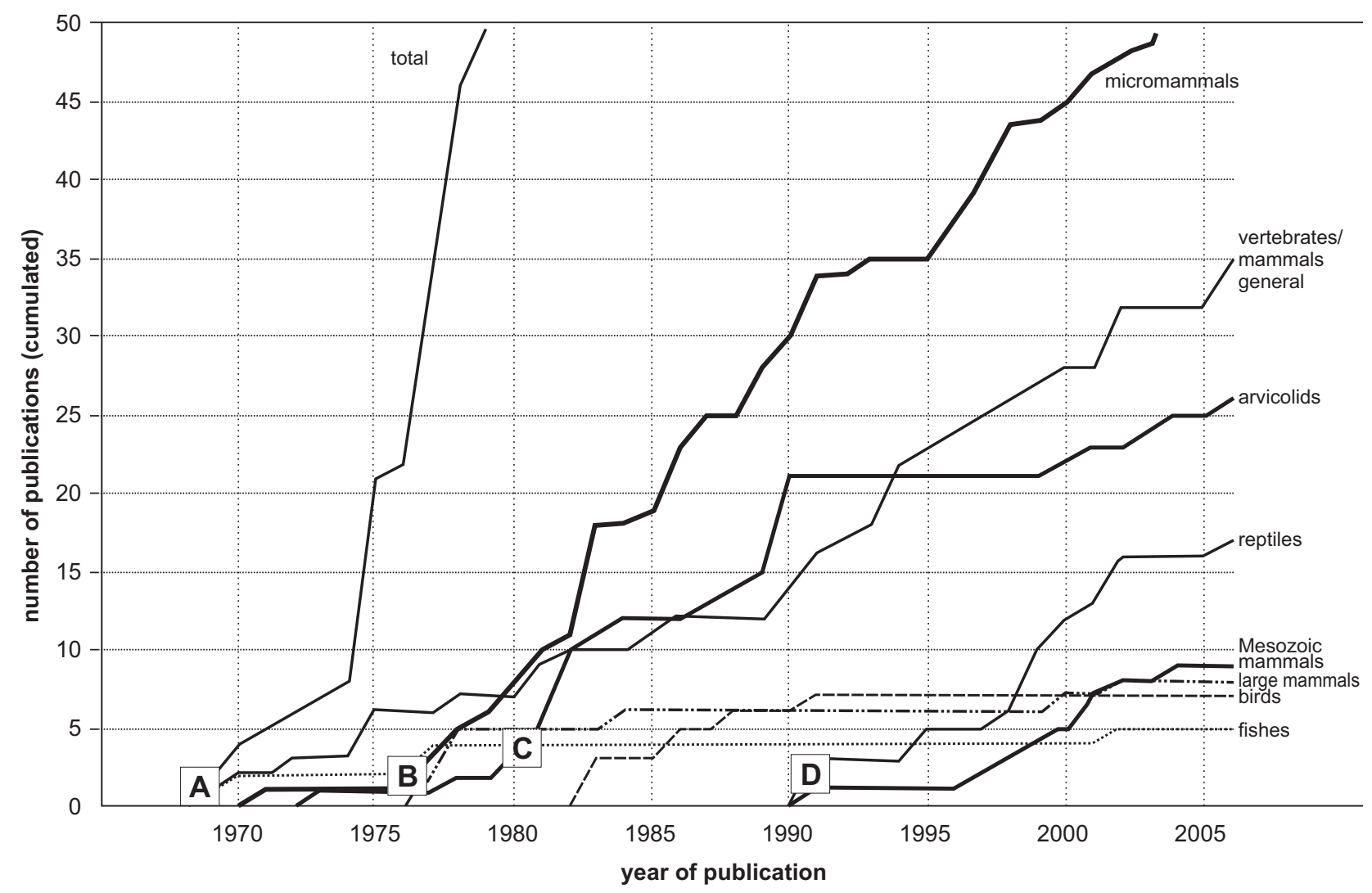

Fig. 4. W.-D. Heinrich's publications as a mirror of his research activities. The graph traces W.-D. Heinrich's publications in terms of the investigated fossil groups. A-D each marks the beginning of a new research field. A - publications on fishes; $\mathbf{B}$ - publications on micromammals; $\mathbf{C}$ - publications on arvicolids; D - publications on Mesozoic mammals and reptiles (Tendaguru projects). Note that the field Quaternary micromammals, especially Arvicolidae, runs through as an unbroken thread. 
mann Jaeger (the new director of this department after the 'Wende' - the political changes in 1989), died unexpectedly. This position required political abilities, which are not normally demanded of a natural scientist 'of the good old type' like WolfDieter Heinrich.

This job has cost him a lot of energy, and this cost continues today, as much that he initiated, shaped and in which he participated, is now disappearing because of the most recent developments in the museum. But, even though he is no longer in the forefront, for the sake of palaeontology and the Museum as a whole, he still offers his opinions and suggestions, and contributes from his long experience.

Thus, Wolf-Dieter Heinrich is highly regarded by his colleagues not only as a first class palaeontologist, but also as a human being in the best possible sense.

\section{List of publications of Wolf-Dieter Heinrich omitting abstracts, book reviews, catalogue ex- tracts etc.}

\section{(in chronological order)}

Heinrich, W.-D. 1969. Fischotolithen aus dem Obermiozän von Hohen Woos. - Geologie 18, Beiheft 67, 111 pp., Berlin.

Heinrich, W.-D. 1969. Wirbeltierfunde aus dem jüngeren Quartär von Pisede bei Malchin (DDR). - Berichte der deutschen Gesellschaft für geologische Wissenschaften A, Geologie und Paläontologie 14 (4): 537-543.

Diebel, K. \& Heinrich, W.-D. 1970. Pisede - eine einmalige Fundstätte quartärer Wirbeltiere in Mecklenburg; Probleme und erste Ergebnisse einer neuen Ausgrabung des Paläontologischen Museums. - Wissenschaftliche Zeitschrift der Humboldt-Universität Berlin, MathematischNaturwissenschaftliche Reihe 19 (2/3): 236-243.

Heinrich, W.-D. 1970. Nachweis der Teleostiergattung Lepidorhombus Günther, 1862 im Chatt von Malliß. - Geologie 19 (7): 883-887.

Peters, G., Heinrich, W.-D., Beurton, P., \& Jäger, K.-D. 1972. Fossile und rezente Dachsbauten mit Massenanreicherungen von Wirbeltierknochen. - Mitteilungen des Zoologischen Museums in Berlin 48 (2): 415-435.

Heinrich, W.-D. \& Jánossy, D. 1973. Lagurus lagurus (Pallas, 1773) (Rodentia, Mammalia) aus dem Jungpleistozän von Burgtonna (Bezirk Erfurt). - Zeitschrift für geologische Wissenschaften 1 (5): 587-592.

Heinrich, W.-D., Jäger, K.-D. \& Gramsch, B. 1974. Quartärpaläontologische Grabungen bei Pisede, Kr. Malchin, mit neolithischen Silexartefaktfunden. - Bodendenkmalpflege in Mecklenburg, Jahrbuch 1974: 49-54.

Cepek, A. G. \& Heinrich, W.-D. 1975. Ein neuer Kleinsäuger-Fundpunkt im Jungmoränengebiet von Buchow-Karpzow (Bezirk Potsdam). - Zeitschrift für geologische Wissenschaften 3 (9): 1235-1248.

Heinrich, W.-D. 1975 (ed.). Die quartäre Wirbeltierfundstätte Pisede bei Malchin (Bez. Neubrandenburg) - Ein Beitrag zur Erd- und Lebensgeschichte des Jung-Pleistozäns und des Holozäns im nordmitteleuropäischen Tiefland, Teil 1. - Wissenschaftliche Zeitschrift der Humboldt-Universität zu Berlin, Mathematisch-Naturwissenschaftliche Reihe 24 (5): 571-716.
Heinrich, W.-D. 1975. Zur Säugetierfauna des ,Rixdorfer Horizontes‘. In Kahlke, H.-D., Cepek, A. G., Erd, K. \& Gellert, J. F. (eds). Exkursionsführer zur Jubiläumstagung 100 Jahre Glazialtheorie im Gebiet der skandinavischen Vereisungen', 3. 11.-7. 11. 1975 in Berlin: 21-23, Gesellschaft für Geologische Wissenschaften der DDR, Berlin.

Heinrich, W.-D. 1975. Anlass und Ablauf der quartärpaläontologischen Ausgrabungen und Untersuchungen bei Pisede (Kr. Malchin). - Wissenschaftliche Zeitschrift der Humboldt-Universität zu Berlin, Mathematisch-Naturwissenschaftliche Reihe 24 (5): 575-578.

Heinrich, W.-D. 1975. Morphographische und strukturelle Kennzeichnung des fossilen Tierbautensystems von Pisede bei Malchin. - Wissenschaftliche Zeitschrift der Humboldt-Universität zu Berlin, Mathematisch-Naturwissenschaftliche Reihe 24 (5): 647-665.

Heinrich, W.-D. 1975. Lithologische Kennzeichnung der Tierbautensedimente von Pisede bei Malchin. - Wissenschaftliche Zeitschrift der Humboldt-Universität $\mathrm{zu}$ Berlin, Mathematisch-Naturwissenschaftliche Reihe 24 (5): 677-687.

Heinrich, W.-D. 1975. Stratigraphische Untersuchungen zur Altersstellung des fossilen Tierbautensystems von Pisede bei Malchin. - Wissenschaftliche Zeitschrift der Humboldt-Universität zu Berlin, Mathematisch-Naturwissenschaftliche Reihe 24 (5): 701-716.

Heinrich, W.-D. \& Jäger, K.-D. 1975. Die Grenzflächen des fossilen Tierbautensystems von Pisede bei Malchin. Wissenschaftliche Zeitschrift der Humboldt-Universität zu Berlin, Mathematisch-Naturwissenschaftliche Reihe 24 (5): 667-676.

Heinrich, W.-D. \& Jäger, K.-D. $1975 .{ }^{14}$ C-Datierungen zur Chronologie des fossilen Tierbautensystems von Pisede bei Malchin. - Wissenschaftliche Zeitschrift der Humboldt-Universität zu Berlin, Mathematisch-Naturwissenschaftliche Reihe 24 (5): 689-692.

Heinrich, W.-D. \& Jäger, K.-D. 1975. Fossile Tierbauten Archive zur jüngsten Erdgeschichte. - Urania 51 (5): $70-73$.

Heinrich, W.-D., Jäger, K.-D. \& Janke, W. 1975. Die jungquartäre Wirbeltierfundstätte Pisede bei Malchin und deren Umgebung als Beispiel einer interdisziplinären landschaftsgeschichtlichen Komplexuntersuchung in der mecklenburgischen Jungmoränenlandschaft. In Die Entwicklung der Territorialstruktur im Nordgebiet der DDR. Exkursionsführer zum Geographenkongress 1975, Teil 1: 57-65, Geographische Gesellschaft der DDR, Neubrandenburg.

Heinrich, W.-D., Krueger, H.-H. \& Wolfschläger, H. 1975. Grabungs- und Bergungsmethodik bei der Untersuchung des fossilen Tierbautensystems von Pisede bei Malchin. Wissenschaftliche Zeitschrift der Humboldt-Universität zu Berlin, Mathematisch-Naturwissenschaftliche Reihe 24 (5): 579-583

Jäger, K.-D. \& Heinrich, W.-D. 1975. Altersbeziehungen zwischen Klüftung, Karbonatumsatz und fossilen Tierbauten im Aufschlussprofil Pisede bei Malchin. - Wissenschaftliche Zeitschrift der Humboldt-Universität zu Berlin, Mathematisch-Naturwissenschaftliche Reihe 24 (5): 693-700.

Janke, W., Jäger, K.-D. \& Heinrich, W.-D. 1975. Geologische und geomorphologische Untersuchungen zur Datierung der quartärpaläontologischen Fundstätte Pisede bei Malchin. - Wissenschaftliche Zeitschrift der Humboldt-Universität zu Berlin, Mathematisch-Naturwissenschaftliche Reihe 24 (5): 593-616.

Heinrich, W.-D. \& Janke, W. 1976. Die jungquartäre Wirbeltierfundstätte Pisede bei Malchin und deren Umgebung als Beispiel einer interdisziplinären landschaftsgeschichtlichen Komplexuntersuchung in der mecklenburgischen Jungmoränenlandschaft. In Die Entwicklung der Territorialstruktur im Nordgebiet der DDR. - Exkursionsführer 
zum Geographenkongress 1975, Teil 1: 57-65, Nachdruck anlässlich der Leopoldina-Exkursion am 22. 6. 1976, Geographische Gesellschaft der DDR, Neubrandenburg.

Fuhrmann, R., Heinrich, W.-D., Mai, D. H. \& Wiegank, F. 1977. Untersuchungen am präelsterkaltzeitlichen Löß von Mahlis (Bezirk Leipzig). - Zeitschrift für geologische Wissenschaften 5 (6): 717-743.

Heinrich, W.-D. 1977 (ed.). Die quartäre Wirbeltierfundstätte Pisede bei Malchin (Bez. Neubrandenburg) - Ein Beitrag zur Erd- und Lebensgeschichte des Jung-Pleistozäns und des Holozäns im nordmitteleuropäischen Tiefland, Teil 2. - Wissenschaftliche Zeitschrift der Humboldt-Universität $\mathrm{zu}$ Berlin, Mathematisch-Naturwissenschaftliche Reihe 26 (3): 225-365.

Heinrich, W.-D. 1977. Die Überlieferung von Fossilien und Lebensspuren im fossilen Tierbautensystem von Pisede bei Malchin. - Wissenschaftliche Zeitschrift der Humboldt-Universität $\mathrm{zu}$ Berlin, Mathematisch-Naturwissenschaftliche Reihe 26 (3): 229-240.

Heinrich, W.-D. 1977. Fischreste aus dem fossilen Tierbautensystem von Pisede bei Malchin. - Wissenschaftliche Zeitschrift der Humboldt-Universität zu Berlin, Mathematisch-Naturwissenschaftliche Reihe 26 (3): 275-281.

Heinrich, W.-D. 1977. Untersuchungen an Vogelresten aus dem fossilen Tierbautensystem von Pisede bei Malchin. Teil 1: Taxonomische und biometrische Kennzeichnung des Fundgutes. - Wissenschaftliche Zeitschrift der Humboldt-Universität zu Berlin, Mathematisch-Naturwissenschaftliche Reihe 26 (3): 329-346.

Heinrich, W.-D. 1977. Untersuchungen an Vogelresten aus dem fossilen Tierbautensystem von Pisede bei Malchin. Teil 2: Paläoökologische und faunengeschichtliche Auswertung des Fundgutes. - Wissenschaftliche Zeitschrift der Humboldt-Universität zu Berlin, Mathematisch-Naturwissenschaftliche Reihe 26 (3): 347-365.

Heinrich, W.-D. \& Jäger, K.-D. 1977. Möglichkeiten und Grenzen der biostratigraphischen Differenzierung fossiler Faunenreste aus quartären Tierbautensystemen mit großer zeitlicher Tiefe. - Wissenschaftliche Zeitschrift der Humboldt-Universität zu Berlin, Mathematisch-Naturwissenschaftliche Reihe 26 (3): 241-247.

Heinrich, W.-D. \& Jánossy, D. 1977. Insektivoren und Rodentier aus dem Travertin von Taubach bei Weimar. - Quartärpaläontologie 2: 401-411.

Ippen, R. \& Heinrich, W.-D. 1977. Pathologische Veränderungen an fossilen Extremitätenknochen von Anuren aus dem fossilen Tierbautensystem von Pisede bei Malchin. Wissenschaftliche Zeitschrift der Humboldt-Universität zu Berlin, Mathematisch-Naturwissenschaftliche Reihe 26 (3): 301-305.

Krolopp, E., Lozek, V., Jäger, K.-D. \& Heinrich, W.-D. 1977. Die Konchylien aus dem fossilen Tierbautensystem von Pisede bei Malchin. - Wissenschaftliche Zeitschrift der Humboldt-Universität Berlin, Mathematisch-Naturwissenschaftliche Reihe 26 (3): 257-274.

Heinrich, W.-D. 1978 (ed.). Das Pleistozän von Burgtonna in Thüringen, Teil II: Die Deckschichtenfolge über dem Travertin von Burgtonna in Thüringen. - Quartärpaläontologie 3: $181-285$.

Heinrich, W.-D. 1978. Biometrische Untersuchungen an fossilen Kleinsäugetieren aus einer jungpleistozänen Deckschichtenfolge über dem interglazialen Travertin von Burgtonna in Thüringen. - Quartärpaläontologie 3: 255-268.

Heinrich, W.-D. 1978. Zum Aussagevermögen fossiler Vogelfunde aus dem küstennahen Tiefland für den Landschaftswandel im Spätglazial der Weichsel-Kaltzeit und im Holozän. - Petermanns Geographische Mitteilungen 122 (3): 149-153.

Heinrich, W.-D. 1978. Zur biometrischen Erfassung eines Evolutionstrends bei Arvicola (Rodentia, Mammalia) aus dem Pleistozän Thüringens. - Säugetierkundliche Informationen 2: 3-21.
Heinrich, W.-D. 1978. Zur Entdeckungs- und Forschungsgeschichte der Wirbeltierfundstätte in der jungpleistozänen Deckschichtenfolge über dem Travertin von Burgtonna in Thüringen. - Quartärpaläontologie 3: 185-186.

Heinrich, W.-D. \& Jäger, K.-D. 1978. Zusammenfassende stratigraphische und palökologische Interpretation des Fossilvorkommens in der untersuchten jungpleistozänen Deckschichtenfolge über dem interglazialen Travertin von Burgtonna in Thüringen. - Quartärpaläontologie 3: 269-285.

Heinrich, W.-D. \& Jánossy, D. 1978. Nachweis von Anas platyrhynchos Linnaeus, 1758 (Anatidae, Aves) aus dem Travertin von Burgtonna in Thüringen. - Quartärpaläontologie 3: 103-105.

Heinrich, W.-D. \& Jánossy, D. 1978. Insektivoren und Rodentier aus dem Travertin von Burgtonna in Thüringen. Quartärpaläontologie 3: 167-170.

Heinrich, W.-D. \& Jánossy, D. 1978. Fossile Vogelreste aus der jungpleistozänen Deckschichtenfolge über dem Travertin von Burgtonna in Thüringen. - Quartärpaläontologie 3: $227-229$.

Heinrich, W.-D. \& Jánossy, D. 1978. Fossile Säugetierreste aus einer jungpleistozänen Deckschichtenfolge über dem interglazialen Travertin von Burgtonna in Thüringen. Quartärpaläontologie 3: 231-254.

Jäger, K.-D. \& Heinrich, W.-D. 1978. Die stratigraphische Gliederung eines fossilführenden jungpleistozänen Deckschichtenprofils über dem Interglazialtravertin von Burgtonna in Thüringen. - Quartärpaläontologie 3: 187-202.

Jäger, K.-D. \& Heinrich, W.-D. 1979. Aktuelle Aspekte und Probleme bei der quartärstratigraphischen Einordnung der mittelpaläolithischen Travertinstation von Ehringsdorf bei Weimar. - Ausgrabungen und Funde 24 (6): 261-267.

Fejfar, O. \& Heinrich, W.-D. 1980. Zur biostratigraphischen Abgrenzung und Gliederung des kontinentalen Quartärs in Europa an Hand von Arvicoliden (Mammalia, Rodentia). - Casopis pro mineralogii a geologii 25 (2): 185-189.

Heinrich, W.-D. 1980. Biostratigraphische Aspekte einer neuen Kleinsäugerfauna aus dem Unteren Travertin von Weimar-Ehringsdorf. - Zeitschrift für geologische Wissenschaften 8 (7): 923-927.

Heinrich, W.-D. 1980. Kleinsäugerfunde aus dem Travertinkomplex von Bilzingsleben. Vorläufige Mitteilung. - Ethnographisch-Archäologische Zeitschrift 21: 36-41.

Fejfar, O. \& Heinrich, W.-D. 1981. Biostratigraphical delimitation and division of the continental Quarternary of Europe on the basis of arvicolids (Rodentia, Mammalia). IUGS-UNESCO, International Geological Correlation Programme, Project 73/1/24 "Quaternary Glaciations in the Northern Hemisphere", Report No. 6: 83-86, Geological Survey, Prag.

Fejfar, O. \& Heinrich, W.-D. 1981. Zur biostratigraphischen Untergliederung des kontinentalen Quartärs in Europa anhand von Arvicoliden (Rodentia, Mammalia). - Eclogae geologiae Helvetiae 74 (3): 997-1006.

Heinrich, W.-D. 1981. Systematische Zusammenstellung der in den thüringischen Interglazialtravertinen von Burgtonna, Taubach und Weimar-Ehringsdorf nachgewiesenen Kleinsäugerarten. - Quartärpaläontologie 4: 127-130.

Heinrich, W.-D. 1981. Fossile Kleinsäugerreste aus dem Unteren Travertin von Weimar-Ehringsdorf (Thüringen, DDR) (Vorläufige Mitteilung). - Quartärpaläontologie 4: 131-143.

Heinrich, W.-D. 1981. Zur stratigraphischen Stellung der Wirbeltierfaunen aus den Travertinfundstätten von WeimarEhringsdorf und Taubach in Thüringen. - Zeitschrift für geologische Wissenschaften 9 (9): 1031-1055.

Jäger, K.-D. \& Heinrich, W.-D. 1981. Correlations between loess stratigraphy and vertebrate palaeontology in the Weichselian of Thuringia and their palaeoecological impli- 
cations. - IUGS-UNESCO, International Geological Correlation Programme, Project 73/1/24 "Quaternary Glaciations in the Northern Hemisphere", Report No. 6: 128-145, Geological Survey, Prag.

Arnold, A., Böhme, G., Fischer, K. \& Heinrich, W.-D. 1982. Eine neue jungpleistozäne Wirbeltierfauna aus Rübeland (Harz). (Vorläufige Mitteilung). - Wissenschaftliche Zeitschrift der Humboldt-Universität zu Berlin, Mathematisch-Naturwissenschaftliche Reihe 31 (3): 169-175.

Fejfar, O. \& Heinrich, W.-D. 1982. A division of the European Quaternary based on arvicolid rodents: A Proposal. - IUGS-UNESCO, International Geological Correlation Programme, Project 73/1/24 "Quaternary Glaciations in the Northern Hemisphere", Report No. 7: 82-83, Geological Survey, Prag.

Fejfar, O. \& Heinrich, W.-D. 1982. Zur Evolution von Mimomys (Rodentia, Mammalia) im Csarnótanum und Villafranchium Europas. - Eclogae geologiae Helvetiae 75 (3): 779-793.

Heinrich, W.-D. 1982. Zur Evolution und Biostratigraphie von Arvicola (Rodentia, Mammalia) im Pleistozän Europas. - Zeitschrift für geologische Wissenschaften 10 (6): 683-735.

Heinrich, W.-D. 1982 (ed.). Wilhelm Otto Dietrich (1881-1964). Gedenkschrift Teil 1. - Zeitschrift für geologische Wissenschaften 10: 883-1057.

Heinrich, W.-D. 1982. Eine altbiharische Kleinsäugerfauna aus Südthüringen. - Zeitschrift für geologische Wissenschaften 10: 923-928.

Heinrich, W.-D. 1982. Ein Evolutionstrend bei Arvicola (Rodentia, Mammalia) und seine Bedeutung für die Biostratigraphie im Pleistozän Europas. - Wissenschaftliche Zeitschrift der Humboldt-Universität zu Berlin, MathematischNaturwissenschaftliche Reihe 31 (3): 155-160.

Heinrich, W.-D. 1982. The importance of Arvicola (Mammalia, Rodentia) for the biostratigraphy of the European Quaternary. - IUGS-UNESCO, International Geological Correlation Programme, Project 73/1/24 "Quaternary Glaciations in the Northern Hemisphere", Report No. 7: 93-96, Geological Survey, Prag.

Jäger, K.-D. \& Heinrich, W.-D. 1982. The travertine at Weimar-Ehringsdorf - An interglacial site of Saalian age? IUGS-UNESCO, International Geological Correlation Programme, Project 73/1/24 "Quaternary Glaciations in the Northern Hemisphere”, Report No. 7: 98-113, Geological Survey, Prag.

Beneš, J., Fischer, K. \& Heinrich, W.-D. 1983. Skelettreste von Raubsäugern (Carnivora, Mammalia) aus dem fossilen Tierbautensystem von Pisede bei Malchin. Teil 1: Taxonomische und biometrische Kennzeichnung des Fundgutes. - Wissenschaftliche Zeitschrift der HumboldtUniversität zu Berlin, Mathematisch-Naturwissenschaftliche Reihe 32 (6): 753-763.

Fejfar, O. \& Heinrich, W.-D. 1983. Arvicoliden-Sukzession und Biostratigraphie des Oberpliozäns und Quartärs in Europa. In Heinrich, W.-D. (ed.). Wirbeltier-Evolution und Faunenwandel im Känozoikum. - Schriftenreihe für geologische Wissenschaften 19/20: 61-109.

Fischer, K. \& Heinrich, W.-D. 1983. Skelettreste von Lepus europaeus Pallas, 1778 (Lagomorpha, Mammalia) aus dem fossilen Tierbautensystem von Pisede bei Malchin. Wissenschaftliche Zeitschrift der Humboldt-Universität zu Berlin, Mathematisch-Naturwissenschaftliche Reihe 32 (6): $713-717$

Fischer, K. \& Heinrich, W.-D. 1983. Skelettreste von Paarhufern (Artiodactyla, Mammalia) aus dem fossilen Tierbautensystem von Pisede bei Malchin. - Wissenschaftliche Zeitschrift der Humboldt-Universität zu Berlin, Mathematisch-Naturwissenschaftliche Reihe 32 (6): $771-776$.

Heinrich, W.-D. 1983 (ed.). Wirbeltier-Evolution und Faunenwandel im Känozoikum. Gedenkschrift für Wilhelm Otto
Dietrich (1881-1964), Teil 2. - Schriftenreihe für geologische Wissenschaften 19/20, 349 pp., Akademie-Verlag, Berlin.

Heinrich, W.-D. 1983 (ed.). Die quartäre Wirbeltierfundstätte Pisede bei Malchin (Bez. Neubrandenburg). - Ein Beitrag zur Erd- und Lebensgeschichte des Jung-Pleistozäns und des Holozäns im nordmitteleuropäischen Tiefland, Teil 3. - Wissenschaftliche Zeitschrift der Humboldt-Universität zu Berlin, Mathematisch-Naturwissenschaftliche Reihe 32 (6): 665-781.

Heinrich, W.-D. 1983. Untersuchungen an Skelettresten von Insectivoren (Insectivora, Mammalia) aus dem fossilen Tierbautensystem von Pisede bei Malchin. Teil 1: Taxonomische und biometrische Kennzeichnung des Fundgutes. Wissenschaftliche Zeitschrift der Humboldt-Universität zu Berlin, Mathematisch-Naturwissenschaftliche Reihe 32 (6): 681-698

Heinrich, W.-D. 1983. Untersuchungen an Skelettresten von Insectivoren (Insectivora, Mammalia) aus dem fossilen Tierbautensystem von Pisede bei Malchin. Teil 2: Paläoökologische und faunengeschichtliche Auswertung des Fundgutes. - Wissenschaftliche Zeitschrift der HumboldtUniversität zu Berlin, Mathematisch-Naturwissenschaftliche Reihe 32 (6): 699-706.

Heinrich, W.-D. 1983. Untersuchungen an Skelettresten von Fledermäusen (Chiroptera, Mammalia) aus dem fossilen Tierbautensystem von Pisede bei Malchin. - Wissenschaftliche Zeitschrift der Humboldt-Universität zu Berlin, Mathematisch-Naturwissenschaftliche Reihe 32 (6): 707-711.

Heinrich, W.-D. 1983. Sciuriden (Rodentia, Mammalia) aus dem fossilen Tierbautensystem von Pisede bei Malchin. Wissenschaftliche Zeitschrift der Humboldt-Universität zu Berlin, Mathematisch-Naturwissenschaftliche Reihe 32 (6): 719-727.

Heinrich, W.-D. 1983. Skelettreste von Raubsäugern (Carnivora, Mammalia) aus dem fossilen Tierbautensystem von Pisede bei Malchin. Teil 2: Paläoökologische und faunengeschichtliche Auswertung. - Wissenschaftliche Zeitschrift der Humboldt-Universität zu Berlin, MathematischNaturwissenschaftliche Reihe 32 (6): 765-770.

Heinrich, W.-D. \& Jäger, K.-D. 1983. Neue Aussagemöglichkeiten der Quartärpaläontologie im Jungmoränengebiet des nördlichen Mitteleuropa. In Kliewe, H., Galon, R., Jäger, K.-D. \& Niewiarowski, W. (eds). Das Jungquartär und seine Nutzung im Küsten- und Binnentiefland der DDR und der VR Polen. - Petermanns Geographische Mitteilungen Ergänzungsheft 282: 184-201.

Heinrich, W.-D. \& Maul, L. 1983. Skelettreste von Nagetieren (Rodentia, Mammalia) aus dem fossilen Tierbautensystem von Pisede bei Malchin. Teil 1: Taxonomische und biometrische Kennzeichnung des Fundgutes. - Wissenschaftliche Zeitschrift der Humboldt-Universität zu Berlin, Mathematisch-Naturwissenschaftliche Reihe 32 (6): 729-743

Heinrich, W.-D. \& Maul, L. 1983. Skelettreste von Nagetieren (Rodentia, Mammalia) aus dem fossilen Tierbautensystem von Pisede bei Malchin. Teil 2: Paläoökologische und faunengeschichtliche Auswertung des Fundgutes. Wissenschaftliche Zeitschrift der Humboldt-Universität zu Berlin, Mathematisch-Naturwissenschaftliche Reihe 32 (6): 745-752.

Heinrich, W.-D., Peters, G., Jäger, K.-D. \& Böhme, G. 1983. Erdbaue von Säugetieren - zusammenfassende Kennzeichnung eines neuen Fundstättentyps im baltischen Vereisungsgebiet. - Wissenschaftliche Zeitschrift der HumboldtUniversität zu Berlin, Mathematisch-Naturwissenschaftliche Reihe 32 (6): 777-781.

Mai, D. H., Mania, D., Nötzold, T., Toepfer, V., Vlcek, E. \& Heinrich, W.-D. 1983 (eds). Bilzingsleben II. Homo erectus - seine Kultur und seine Umwelt. - Veröffentlichungen des Landesmuseum für Vorgeschichte Halle 36, 258 pp., Verlag der Wissenschaften, Berlin. 
Heinrich, W.-D. 1984. Accipiter nisus (Linnaeus) aus dem Travertin der Weimarer Parkhöhlen. - Quartärpaläontologie 5: 349-350.

Heinrich, W.-D. 1984. Arvicola sp. (Mammalia, Rodentia) aus dem Pleistozän der Parkhöhlen in Weimar. - Quartärpaläontologie 5: 431-432.

Heinrich, W.-D. 1984. Die quartäre Wirbeltierfundstätte Pisede bei Malchin (Bez. Neubrandenburg) - Ein Beitrag zur Erd- und Lebensgeschichte des Jung-Pleistozäns und des Holozäns im nordmitteleuropäischen Tiefland. Teil III. - Humboldt-Universität, Jahrgang 28 (25): 6.

Jäger, K.-D., Heinrich, W.-D., Hrabowski, K., Schäfer, D. \& Weber, T. 1984. XI. Kongreß der INQUA (International Union for Quaternary Research), Moskau 1982. Ethnographisch-Archäologische Zeitschrift 25 (3): $553-$ 558.

Fejfar, O. \& Heinrich, W.-D. 1985. Zur Bedeutung der Wirbeltierfundstätten von Ivanovce und Hajnácka für die Säugetierpaläontologie im Pliozän und frühen Pleistozän in Europa: Kenntnisstand und Probleme. - Věstník Ústřednǐho ústavu geologického 60 (4): 213-224.

Heinrich, W.-D. 1985. Zur Erforschung von fossilen Kleinsäugerfaunen aus dem Eiszeitalter im Gebiet der DDR Stand und Probleme. - Säugetierkundliche Informationen 2 (9): 203-226.

Heinrich, W.-D. 1986. Funde des Waldelefanten (Palaeoloxodon antiquus) im Eiszeitalter. - Sozialistische Forstwirtschaft 1986 (2): 3.

Heinrich, W.-D. 1986. Zur faunen- und landschaftsgeschichtlichen Aussage eines Fundes vom Eiszeitziesel, Citellus (Colobotis) superciliosus, aus Mecklenburg. - Acta Universitatis Nicolai Copernici, Geografia 21: 103-108.

Heinrich, W.-D. 1986. Zur Geschichte der Erforschung von fossilen Kleinsäugerfaunen aus Interglazialtravertinen in Thüringen. - Quartärpaläontologie 6: 67-74.

Heinrich, W.-D., Fejfar, O. \& Schäfer, D. 1986. Nachweis eines Otters (Mammalia, Carnivora) in der paläolithischen Travertinfundstätte von Weimar-Ehringsdorf in Thüringen. - Ausgrabungen und Funde 31 (5): 199-203.

Fejfar, O. \& Heinrich, W.-D. 1987. Zur biostratigraphischen Gliederung des jüngeren Känozoikums in Europa an Hand von Muriden und Cricetiden (Rodentia, Mammalia). - Časopis pro mineralogii a geologii 32 (1): 1-16.

Heinrich, W.-D. 1987. Neue Ergebnisse zur Evolution und Biostratigraphie von Arvicola (Rodentia, Mammalia) im Quartär Europas. - Zeitschrift für geologische Wissenschaften 15 (3): 389-406.

Heinrich, W.-D. \& Jäger, K.-D. 1987. Paläoökologische und landschaftsgeschichtliche Interpretation einer klinalen Merkmalsvariation bei rezenten und fossilen Populationen von Crocidura leucodon (Mammalia: Soricidae). - Petermanns Geographische Mitteilungen 131 (2): 93-98.

Fejfar, O. \& Heinrich, W.-D. 1988. Report on the First International Meeting "Evolution, Phylogeny, and Biostratigraphy of Arvicolids (Rodentia, Mammalia)", Rohanov, Czechoslovakia, May 20-29, 1987. - Bulletin of the Society of Vertebrate Paleontology, 1988: 11-12, Los Angeles.

Heinrich, W.-D. \& Fejfar, O. 1988. Fund eines Lutrinen (Mammalia: Carnivora, Mustelidae) aus dem Unteren Travertin von Weimar-Ehringsdorf in Thüringen. - Zeitschrift für geologische Wissenschaften 16 (6): 515-529.

Fejfar, O. \& Heinrich, W.-D. 1989. Biostratigraphical subdivision of the European Late Cenozoic based on muroid rodents (Mammalia). - Memorie della Società Geologica Italiana 31: 185-190.

Fejfar, O., Heinrich, W.-D. \& Jäger, K.-D. 1989. First International Meeting "Evolution, Phylogeny and Biostratigraphy of Arvicolids (Rodentia, Mammalia", Rohanov/CSSR 1987. - Ethnographisch-Archäologische Zeitschrift 30 (1): $176-177$.

Heinrich, W.-D. 1989. Biometrische Untersuchungen an Fossilresten des Bibers (Castor fiber L.) aus thüringischen
Travertinen. - Ethnographisch-Archäologische Zeitschrift 30 (2): 394-403.

Heinrich, W.-D. 1989. Biostratigraphische Untersuchungen an fossilen Kleinsäugerresten aus dem Travertin von Bilzingsleben. - Ethnographisch-Archäologische Zeitschrift 30 (2): 379-393

Heinrich, W.-D. 1989. Hans-Dietrich Kahlke zum 65. Geburtstag. - Ethnographisch-Archäologische Zeitschrift 30 (2): 339-340.

Benecke, N., Böhme, G. \& Heinrich, W.-D. 1990. Wirbeltierreste aus interglazialen Beckensedimenten von Gröbern (Kr. Gräfenhainichen) und Grabschütz (Kr. Delitzsch). Altenburger naturwissenschaftliche Forschungen 5: 231-281.

Fejfar, O. \& Heinrich, W.-D. 1990 (eds). International Symposium "Evolution, Phylogeny, Biostratigraphy of Arvicolids (Rodentia, Mammalia)", Rohanov (Czechoslovakia) May 1987. 448 pp., Pfeil, Munich.

Fejfar, O. \& Heinrich, W.-D. 1990. International symposium "Evolution, Phylogeny and Biostratigraphy of fossil Arvicolids", Rohanov, May 1987: Topics of the meeting and results of the discussions. In Fejfar, O. \& Heinrich, W.-D. (eds). International Symposium "Evolution, Phylogeny, and Biostratigraphy of Arvicolids (Rodentia, Mammalia)", Rohanov (Czechoslovakia) May 1987: 443-448, Pfeil, Munich.

Fejfar, O. \& Heinrich, W.-D. 1990. Muroid rodent biochronology of the Neogene and Quaternary in Europe. In Lindsay, E. H., Fahlbusch, V. \& Mein, P. (eds). European Neogene Mammal Chronology: 91-117, Plenum Press, New York.

Fejfar, O. \& Heinrich, W.-D. 1990. Proposed biostratigraphic division of the European continental Neogene and Quaternary based on muroid rodents (Rodentia: Mammalia). In Fejfar, O. \& Heinrich, W.-D. (eds). International Symposium "Evolution, Phylogeny, and Biostratigraphy of Arvicolids (Rodentia, Mammalia)", Rohanov (Czechoslovakia) May 1987: 115-124, Pfeil, Munich.

Fejfar, O., Heinrich, W.-D. \& Heintz, E. 1990. Neues aus dem Villafranchium von Hajnacka bei Filakovo (Slowakei, CSSR). - Quartärpaläontologie 8: 47-70.

Heinrich, W.-D. 1990. Nachweis von Lagurus lagurus (Pallas, 1773) für das Pleistozän von Neumark-Nord, Kreis Merseburg. In Mania, D., Thomae, M., Litt, T. \& Weber, T. (eds). Neumark-Gröbern - Beiträge zur Jagd des mittelpaläolithischen Menschen. - Veröffentlichungen des Landesmuseums für Vorgeschichte Halle 43: $167-175$.

Heinrich, W.-D. 1990. Some aspects of evolution and biostratigraphy of Arvicola (Rodentia, Mammalia) in the Central European Pleistocene. In Fejfar, O. \& Heinrich, W.-D. (eds). International Symposium "Evolution, Phylogeny, and Biostratigraphy of Arvicolids (Rodentia, Mammalia)", Rohanov (Czechoslovakia) May 1987: 165-182, Pfeil, Munich.

Heinrich, W.-D. 1990. Review of fossil arvicolids (Mammalia, Rodentia) from the Pliocene and Quaternary in the German Democratic Republic. In Fejfar, O. \& Heinrich, W.-D. (eds). International Symposium "Evolution, Phylogeny, and Biostratigraphy of Arvicolids (Rodentia, Mammalia)", Rohanov (Czechoslovakia) May 1987: 183-200, Pfeil, Munich.

Repenning, C. A., Fejfar, O. \& Heinrich, W.-D. 1990. Arvicolid rodent biochronology of the Northern Hemisphere. In Fejfar, O. \& Heinrich, W.-D. (eds). International Symposium "Evolution, Phylogeny, and Biostratigraphy of Arvicolids (Rodentia, Mammalia)", Rohanov (Czechoslovakia) May 1987, 385-418, Pfeil, Munich.

Bloos, G., Böttcher, R., Heinrich, W.-D. \& Münzing, K. 1991. Ein Vorkommen von Kleinvertebraten in jungpleistozänen Deckschichten (Wende Eem/Würm) bei Steinheim an der Murr. - Stuttgarter Beiträge zur Naturkunde, Serie B (Geologie und Paläontologie) 170: 1-72. 
Fischer, K., Guenther, E. W., Heinrich, W.-D., Mania, D., Musil, R. \& Nötzold, T. 1991 (eds). Bilzingsleben IV. Homo erectus - seine Kultur und seine Umwelt. - Veröffentlichungen des Landesmuseum für Vorgeschichte Halle, 44, 248 pp., Verlag der Wissenschaften, Berlin.

Fischer, K. \& Heinrich, W.-D. 1991. Sus scrofa L. (Mammalia: Artiodactyla, Suidae) aus der altpaläolithischen Fundstätte Bilzingsleben in Thüringen. In Fischer, K., Guenther, E. W., Heinrich, W.-D., Mania, D., Musil, R. \& Nötzold, T. (eds). Bilzingsleben IV. Homo erectus seine Kultur und seine Umwelt. - Veröffentlichungen des Landesmuseum für Vorgeschichte Halle, 44: 131-138.

Heinrich, W.-D. 1991. Biometrische Untersuchungen an Fossilresten des Bibers (Castor fiber L.) aus der Fundstätte Bilzingsleben. In Fischer, K., Guenther, E.W., Heinrich, W.-D., Mania, D., Musil, R. \& Nötzold, T. (eds). Bilzingsleben IV. Homo erectus - seine Kultur und seine Umwelt. - Veröffentlichungen des Landesmuseum für Vorgeschichte Halle, 44: 35-62.

Heinrich, W.-D. 1991. Die Kleinsäugerfauna des Eem-Interglazials von Schönfeld, Kr. Calau (Niederlausitz). - Natur und Landschaft in der Niederlausitz 1991, Sonderheft: Eem von Schönfeld: 177-188.

Heinrich, W.-D. 1991. Paläoökologische und biostratigraphische Kennzeichnung der pleistozänen Säugetierfaunen von Schönfeld, Kr. Calau, in der Niederlausitz. - Natur und Landschaft in der Niederlausitz, 1991, Sonderheft: Eem von Schönfeld: 190-199.

Heinrich, W.-D. 1991. Über Brancatherulum tendagurense Dietrich, 1927 (Mammalia: Eupantotheria) aus dem Oberjura von Tendaguru, Tansania. Eine vorläufige Mitteilung. Mitteilungen aus dem Zoologischen Museum in Berlin 67 (1): 97-104.

Heinrich, W.-D. 1991. Zur biostratigraphischen Einordnung der Fundstätte Bilzingsleben an Hand fossiler Kleínsäugetiere. In Fischer, K., Guenther, E. W., Heinrich, W.-D., Mania, D., Musil, R. \& Nötzold, T. (eds). Bilzingsleben IV. Homo erectus - seine Kultur und seine Umwelt. Veröffentlichungen des Landesmuseum für Vorgeschichte Halle, 44: 71-79.

Heinrich; W.-D. 1992. Zur Quartärstratigraphie und Säugetierpaläontologie im südlichen Randbereich der skandinavischen Inlandvergletscherungen. In Billwitz, K., Jäger, K.-D. \& Janke, W. (eds). Jungquartäre Landschaftsräume. Aktuelle Forschungen zwischen Atlantik und Tienschan: 71-80, Springer, Berlin.

Heinrich, W.-D. 1993. Zur Paläoökologie und Biostratigraphie der fossilen Kleinsäugetiere aus dem Travertinkomplex von Bilzingsleben. - Ethnographisch-Archäologische Zeitschrift 34: 583-591.

Kirsche, W., Richter, W. \& Heinrich, W.-D. 1993. Zur Mikrostruktur fossiler Säugetierknochen aus dem Rixdorfer Horizont der Weichsel-Kaltzeit von Niederlehme bei Königs Wusterhausen (Brandenburg). - Annals of Anatomy 175: $177-184$.

Böhme, G. \& Heinrich, W.-D. 1994. Zwei neue Wirbeltierfaunen aus der pleistozänen Schichtenfolge des Travertins von Weimar-Ehringsdorf. Vorläufige Mitteilung. - Ethnographisch-Archäologische Zeitschrift 35 (1): 67-74.

Heinrich, W.-D. 1994. Biostratigraphische Aussagen der Säugetierpaläontologie zur Altersstellung pleistozäner Travertinfundstätten in Thüringen. - Berliner geowissenschaftliche Abhandlungen E13 (B. Krebs-Festschrift): 251-267.

Gunga, H.-Chr., Kirsch, K. A., Baartz, F., Röcker, L., Heinrich, W.-D., Lisowski, W., Wiedemann, A. \& Albertz, J. 1995. New data on the dimensions of Brachiosaurus brancai and their physiological implications. - Naturwissenschaften 82 (4): 190-192.

Koenigswald, W. von \& Heinrich, W.-D. 1996. Kurze Charakterisierung der Veränderungen in der Säugetierfauna des Jungquartärs in Mitteleuropa. - Tübinger Monographien zur Urgeschichte 11 (H. Müller-Beck-Festschrift): 437448.

Fejfar, O., Heinrich, W.-D. Pevzner, M. A. \& Vangenheim, E. A. 1997. Late Cenozoic sequences of mammalian sites in Eurasia: an updated correlation. - Palaeogeography, Palaeoclimatology, Palaeoecology 133 (3/4): 259-288.

Heinrich, W.-D. 1997. Zur Taphonomie, Paläoökologie und Biostratigraphie fossiler Kleinsäugerfaunen aus dem mittelpleistozänen Travertinkomplex Bilzingsleben II in Thüringen. In Mania, D., Mania, U., Heinrich, W.-D., Fischer, K., Böhme, G., Turner, A., Erd, K. \& Mai, D. H. (eds). Bilzingsleben V. Homo erectus - seine Kultur und Umwelt: 121-134, 256-259, Verlag Ausbildung + Wissen, Bad Homburg.

Heinrich, W.-D. 1997. Über Trogontherium cuvieri (Mammalia, Rodentia: Castoridae) aus dem mittelpleistozänen Travertinkomplex Bilzingsleben II in Thüringen. In Mania, D., Mania, U., Heinrich, W.-D., Fischer, K., Böhme, G., Turner, A., Erd, K. \& Mai, D. H. (eds). Bilzingsleben V. Homo erectus - seine Kultur und Umwelt: 135-182, 259-260, Verlag Ausbildung + Wissen, Bad Homburg.

Mania, D., Mania, U., Heinrich, W.-D., Fischer, K., Böhme, G., Turner, A., Erd, K. \& Mai, D. H. 1997 (eds). Bilzingsleben V. Homo erectus - seine Kultur und Umwelt. 264 pp., Verlag Ausbildung + Wissen, Bad Homburg.

Böhme, G. \& Heinrich, W.-D. 1998. Neue Wirbeltierfunde aus den altpleistozänen Flußablagerungen von Kalbsrieth an der Unstrut. - Praehistoria Thuringica, 2: 73-88.

Christian, A. \& Heinrich, W.-D. 1998. The neck posture of Brachiosaurus brancai. - Mitteilungen aus dem Museum für Naturkunde in Berlin, Geowissenschaftliche Reihe 1: $73-80$.

Fejfar, O, Heinrich, W.-D. \& Lindsay, E. H. 1998. Updating the European Neogene rodent biochronology. In Aguilar, J.-P., Legendre, S. \& Michaux, J. (eds). Actes du Congrés BiochroM'97. - Mémoires et Travaux de l'Institut de Montpellier de l'École Pratique des Hautes Études 21: 563-565.

Fejfar, O, Heinrich, W.-D. \& Lindsay, E. H. 1998. Updating the Neogene rodent biochronology in Europe. In Kolfschoten, Th. van \& Gibbard, P. L. (eds). The Dawn of the Quaternary, Mededelingen Nederlands Instituut voor Toegepaste Geowetenschappen TNO 60: 533-553, Haarlem.

Heinrich, W.-D. 1998. Evolutionary trends in Trogontherium cuvieri (Mammalia, Rodentia, Castoridae) and their implications. In Kolfschoten, Th. van \& Gibbard, P. L. (eds). The Dawn of the Quaternary, Mededelingen Nederlands Instituut voor Toegepaste Geowetenschappen TNO 60: 573-578, Haarlem.

Heinrich, W.-D. 1998. Late Jurassic mammals from Tendaguru, Tanzania, East Africa. - Journal of Mammalian Evolution 5 (4): 269-290.

Heinrich, W.-D. 1998. Weitere Funde von Kleinsäugetieren aus dem Travertinkomplex Bilzingsleben II in Thüringen. Praehistoria Thuringica 2: 89-95.

Christian, A., Heinrich, W.-D. \& Golder, W. 1999. Posture and mechanics of the forelimbs of Brachiosaurus brancai (Dinosauria: Sauropoda). - Mitteilungen aus dem Museum für Naturkunde in Berlin, Geowissenschaftliche Reihe 2: 63-73.

Gunga, H.-Chr., Kirsch, K., Rittweger, J., Clarke, A., Albertz, J., Wiedemann A., Mokry, S., Suthau, T., Wehr, A., Heinrich, W.-D. \& Schultze, H.-P. 1999. Body size and body volume distribution in two sauropods from the Upper Jurassic of Tendaguru/Tanzania (East Africa). - Mitteilungen aus dem Museum für Naturkunde in Berlin, Geowissenschaftliche Reihe 2: 91-102.

Heinrich, W.-D. 1999. The taphonomy of dinosaurs from the Upper Jurassic of Tendaguru, Tanzania (East Africa), based on field sketches of the German Tendaguru expedition (1909-1913). - Mitteilungen aus dem Museum für 
Naturkunde in Berlin, Geowissenschaftliche Reihe 2: 25-61.

Heinrich, W.-D. 1999. First haramiyid (Mammalia, Allotheria) from the Mesozoic of Gondwana. - Mitteilungen aus dem Museum für Naturkunde in Berlin, Geowissenschaftliche Reihe 2: 159-170.

Koenigswald, W. von \& Heinrich, W.-D. 1999. Mittelpleistozäne Säugetierfaunen aus Mitteleuropa - der Versuch einer biostratigraphischen Zuordnung. - Kaupia 9: 53-112.

Unwin, D. M. \& Heinrich, W.-D. 1999. On a pterosaur jaw from the Upper Jurassic of Tendaguru, Tanzania. - Mitteilungen aus dem Museum für Naturkunde in Berlin, Geowissenschaftliche Reihe 2: 121-134.

Bonaparte, J. F., Heinrich, W.-D. \& Wild, R. 2000. Review of Janenschia Wild, with the description of a new sauropod from the Tendaguru beds of Tanzania and a discussion on the systematic value of procoelus caudal vertebrae in the sauropoda. - Palaeontographica Abteilung A 256 (1-3): 25-76.

Heinrich, W.-D. 2000. Rodentier-Biostratigraphie und Altersstellung der mittelpleistozänen Homo erectus-Schichten der Travertinfundstätte Bilzingsleben II in Thüringen, Mitteldeutschland. - Praehistoria Thuringica 4: $28-40$.

Maul, L. C., Rekovets, L., Heinrich, W.-D., Keller, Th. \& Storch, G. 2000. Arvicola mosbachensis (Schmidtgen 1911) of Mosbach 2: a basic sample for the early evolution of the genus and a reference for further biostratigraphical studies. - Senckenbergiana lethaea 80 (1): 129-147.

Heinrich, W.-D. 2001. Kleinsäugerreste aus interglazialen Ablagerungen von Neumark-Nord, Mitteldeutschland. Vorläufige Mitteilung. - Praehistoria Thuringica 6/7: $132-138$

Heinrich, W.-D. 2001. New records of Staffia aenigmatica (Mammalia, Allotheria, Haramiyida) from the Upper Jurassic of Tendaguru in southeastern Tanzania, East Africa. Mitteilungen aus dem Museum für Naturkunde in Berlin, Geowissenschaftliche Reihe 4: 239-255.

Heinrich, W.-D. 2001. Erster Nachweis von Lagurus lagurus (Pallas, 1773) (Mammalia, Rodentia, Arvicolidae) für das Jungpleistozän Norddeutschlands. - Lynx, n. s. 32 (O. FejfarFestschrift): 247-253.

Heinrich, W.-D. 2001. Im Schatten der Dinosaurier. Säugetiere aus der späten Jura-Zeit Ostafrikas. - Theatrum naturae 1: 12-13.

Heinrich, W.-D. 2001. Dinosaurierjagd in Ostafrika: Die Deutsche Tendaguru-Expedition 1909-1913. - Theatrum naturae 1: 20-22.

Heinrich, W.-D., Bussert, R., Aberhan, M., Hampe, O., Kapilima, S., Schrank, E., Schultka, St., Maier, G., Msaky, E., Sames, B., Chami, R. 2001. The German-Tanzanian Tendaguru Expedition 2000. - Mitteilungen aus dem Museum für Naturkunde in Berlin, Geowissenschaftliche Reihe 4: 223-237.

Heinrich, W.-D. \& Schultze, H.-P. 2001. Weltnaturerbe Grube Messel. Das Land Hessen zeigt Funde aus dem Pompeji der Paläontologie. - Theatrum naturae 1: 26-29.

Aberhan, M., Bussert, R., Heinrich, W.-D., Schrank, E., Schultka, St., Sames, B., Kriwet, J. \& Kapilima, S. 2002. Palaeoecology and depositional environments of the Tendaguru Beds (Late Jurassic to Early Cretaceous, Tanzania). - Mitteilungen aus dem Museum für Naturkunde in Berlin, Geowissenschaftliche Reihe 5: 17-42.

Aberhan, M., Heinrich, W.-D. \& Schultka, St. 2002 (eds). Festschrift for Professor Hans-Peter Schultze on his 65th birthday. With 20 contributions from 41 authors. - Mitteilungen aus dem Museum für Naturkunde in Berlin, Geowissenschaftliche Reihe 5, $384 \mathrm{~S}$.

Arratia, G., Kriwet, J. \& Heinrich, W.-D. 2002. Selachians and Actinopterygians from the Upper Jurassic of Tendaguru, Tanzania. - Mitteilungen aus dem Museum für
Naturkunde in Berlin, Geowissenschaftliche Reihe 5: 205-228.

Gunga, H.-Chr., Kirsch, K., Rittweger, J., Clarke, A., Albertz, J., Wiedemann, A., Wehr, A., Heinrich, W.-D. \& Schultze, H.-P. 2002. Dimensions of Brachiosaurus brancai, Dicraeosaurus hansemanni and Diplodocus carnegii and their implications for gravitational physiology. In Moravec, J., Takeda, N. \& Singal, P.K. (eds). Adaptation Biology and Medicine (volume 3): 156-169, Narosa Publishing House, New Delhi.

Heinrich, W.-D. 2002. Der Rixdorfer Horizont - ein Fundstättentyp eiszeitlicher Säugetiere in Berlin und Brandenburg. - Humboldt Spektrum 9 (2/3): 70-75.

Heinrich, W.-D., Arratia, G. \& Kriwet, J. 2002. Late Jurassic Vertebrates from Tendaguru, Tanzania. - Journal of Vertebrate Paleontology Supplement 22 (3): 63-64A.

Heinrich, W.-D. \& Hermsdorf, N. 2002. Jungpleistozäne Kleinsäugerreste von Zachow bei Ketzin in Brandenburg. Vorläufige Mitteilung. - Brandenburgische Geowissenschaftliche Beiträge 9 (1/2): 117-122.

Heinrich, W.-D. \& Jaeger, H. 2002. The ancestors of mammals. In Museum of Natural History: A guide through the museum: 24-27, Museum für Naturkunde, Berlin.

Burdukiewicz, M., Fiedler, L., Heinrich, W.-D., Justus, A. \& Brühl, E. 2003 (eds). Erkenntnisjäger. Kultur und Umwelt des frühen Menschen. Festschrift für Dietrich Mania. Mit 63 Beiträgen von 78 Autoren. - Veröffentlichungen des Landesamtes für Archäologie Sachsen-Anhalt 57 (1/2): $1-713$.

Heinrich, W.-D. 2003. Expeditionen in die Erdgeschichte Ostafrikas: Die Dinosaurierlagerstätte Tendaguru in Tansania. - Sitzungsberichte der Gesellschaft Naturforschender Freunde zu Berlin (N. F.) 42: 5-30.

Heinrich, W.-D. 2003. Rodentier-Biostratigraphie und Altersstellung pleistozäner Säugetier-Fundstätten Mitteldeutschlands. - Veröffentlichungen des Landesamtes für Archäologie Sachsen-Anhalt 57 (I): 237-245.

Heinrich, W.-D. \& Hermsdorf, N. 2003. Lemmus lemmus (Linnaeus, 1758) und Dicrostonyx gulielmi (Sandford, 1870) (Mammalia, Rodentia) aus dem weichselzeitlichen Rixdorfer Horizont von Niederlehme bei Königs Wusterhausen in Brandenburg. - Brandenburgische Geowissenschaftliche Beiträge 10 (1/2): 153-158.

Mania, D., Altermann, M., Böhme, G., Erd, K., Fischer, K., Heinrich, W.-D., Kremenetzki, C., Made, J. van der, Mai, D.-H., Musil, R., Pietrzeniuk, E., Schüler, T., Vlcek, E. \& Steiner, W. 2003. Die Travertine in Thüringen und im Harzvorland. - Hallesches Jahrbuch für Geowissenschaften, Reihe B: Geologie, Paläontologie, Mineralogie, Beiheft 17: 1-82.

Heinrich, W.-D. 2004. Nachweis von Lagurus lagurus (Pallas, 1773) im archäologischen Fundhorizont der Travertinfundstätte Bilzingsleben II. - Praehistoria Thuringica 10: $16-21$.

Heinrich, W.-D. 2004. Nomenclatorial note: Allostaffia, a new genus name for Staffia Heinrich, 1999 (Mammalia, Allotheria, Haramiyida) preoccupied by Staffia Schubert 1912 (Foraminifera). - Mitteilungen aus dem Museum für Naturkunde in Berlin, Geowissenschaftliche Reihe 7: 153.

Heinrich, W.-D. 2004. Zur Taphonomie, Paläoökologie und Biostratigraphie fossiler Kleinsäugerfaunen aus dem mittelpleistozänen Travertinkomplex Bilzingsleben II in Thüringen. In Mania, D., Mania, U., Heinrich, W.-D., Fischer, K., Böhme, G., Turner, A., Erd, K. \& Mai, D. H. (eds). Bilzingsleben V. Homo erectus - seine Kultur und Umwelt, 2.Aufl.: 121-134, 256-259, Beier \& Beran, Langenweissbach.

Heinrich, W.-D. 2004. Über Trogontherium cuvieri (Mammalia, Rodentia: Castoridae) aus dem mittelpleistozänen Travertinkomplex Bilzingsleben II. In Mania, D., Mania, U., Heinrich, W.-D., Fischer, K., Böhme, G., Turner, A., Erd, K. \& Mai, D. H. (eds). Bilzingsleben V. Homo erec- 
tus - seine Kultur und Umwelt, 2. Aufl.: 135-182, 259-260, Beier \& Beran, Langenweissbach.

Heinrich, W.-D. 2004. Nagetiere (Mammalia: Rodentia) aus dem Eem-Interglazial von Phöben bei Werder, Brandenburg. - Brandenburgische Geowissenschaftliche Beiträge $11(1 / 2)$ : 169-172.

Mania, D., Mania, U., Heinrich, W.-D., Fischer, K., Böhme, G., Turner, A., Erd, K. \& Mai, D. H. 2004 (eds). Bilzingsleben V. Homo erectus - seine Kultur und Umwelt, 2. Aufl. 264 pp., Beier \& Beran, Langenweissbach.

Fejfar, O., Heinrich, W.-D., Kordos, L. \& Lindsay, E. 2005. Miklos Kretzoi (1907-2005). - Society of Vertebrate Paleontology, News Bulletin 198: 26-29.

Heinrich, W.-D. \& Hermsdorf, N. 2005. Erster Nachweis eines Desmans, Desmana moschata (Linnaeus, 1758) (Mammalia: Insectivora), im Pleistozän der Mark Brandenburg. - Brandenburgische Geologische Beiträge 12 $(1 / 2): 55-60$.

Dorka, M. \& Heinrich, W.-D. 2006. Tetrapod teeth from a Rhaetian (Upper Triassic) bonebed near Friedland (NWGermany). - Palaeontographica Abteilung A (W. von Koenigswald-Festschrift) 278 (1-6): 1-13.

\section{In press}

Heinrich, W.-D. Arvicola cf. cantianus (Mammalia: Rodentia, Arvicolidae) aus den pleistozänen Beckenablagerungen von Klinge bei Cottbus (Brandenburg). - Natur und Landschaft in der Niederlausitz, Cottbus.

Heinrich, W.-D. Castor fiber Linnaeus, 1758 (Mammalia: Rodentia, Castoridae) aus den pleistozänen Beckenablagerungen von Klinge bei Cottbus (Brandenburg). - Natur und Landschaft in der Niederlausitz, Cottbus.

Heinrich, W.-D. Kleinsäugerfunde aus dem Oberen Travertin von Weimar-Ehringsdorf. - Praehistoria Thuringica, Artern.

Heinrich, W.-D. Kleinsäugerreste aus den pleistozänen Beckenablagerungen von Neumark-Nord 1, Mitteldeutschland. Veröffentlichungen des Landesamtes für Archäologie Sachsen-Anhalt, Halle (Saale).

Maul, L. C. \& Heinrich, W.-D. Neue Daten zur Kleinsäugerbiostratigraphie und Altersabfolge deutscher Quartärfundstellen. - K.-D. Jäger-Festschrift, Weimar.

Schäfer, D., Heinrich, W.-D., Böhme, G. \& Steiner, W. Aspects of the geology, palaeontology and archaeology of the travertine site of Weimar-Ehringsdorf (Thuringia, Central Europe). - Courier Forschungsinstitut Senckenberg (H.-D. Kahlke-Festschrift), Frankfurt a. M. 